\title{
Forward solvers for photon migration in the presence of highly and totally absorbing objects embedded inside diffusive media
}

\author{
Angelo Sassaroli, ${ }^{1}$ Antonio Pifferi, ${ }^{2}$ Davide Contini, ${ }^{2}$ Alessandro Torricelli, ${ }^{2}$ Lorenzo Spinelli, ${ }^{3}$ \\ Heidrun Wabnitz, ${ }^{4}$ Paola Di Ninni, ${ }^{5}$ Giovanni Zaccanti, ${ }^{5}$ and Fabrizio Martelli ${ }^{5, *}$ \\ ${ }^{1}$ Department of Biomedical Engineering, Tufts University, 4 Colby St., Medford, Massachusetts 02155, USA \\ ${ }^{2}$ Dipartimento di Fisica, Politecnico di Milano, Piazza Leonardo da Vinci 32, 20133 Milano, Italy \\ ${ }^{3}$ Istituto di Fotonica e Nanotecnologie, Consiglio Nazionale delle Ricerche, \\ Piazza Leonardo da Vinci 32, 20133 Milano, Italy \\ ${ }^{4}$ Physikalisch-Technische Bundesanstalt (PTB), Abbestr. 2-12, 10587 Berlin, Germany \\ ${ }^{5}$ Dipartimento di Fisica e Astronomia dell'Università degli Studi di Firenze, \\ Via G. Sansone 1, 50019 Sesto Fiorentino, Italy \\ *Corresponding author: fabrizio.martelli@unifi.it
}

Received October 4, 2013; revised December 13, 2013; accepted December 13, 2013;

posted December 24, 2013 (Doc. ID 198723); published February 5, 2014

\begin{abstract}
In this paper, after a critical review of the literature, we present two forward solvers and a new methodology for description of photon migration in the presence of totally absorbing inclusions embedded in diffusive media in both time and CW domains. The first forward solver is a heuristic approach based on a higher order perturbation theory applied to the diffusion equation (DE) [denoted eighth-order perturbation theory (EOPT)]. The second forward solver [denoted eighth-order perturbation theory with the equivalence relation (EOPTER) ] is obtained by combining the EOPT solver with the adoption of the equivalence relation (ER) [J. Biomed. Opt. 18, 066014 (2013)]. These forward solvers can possibly overcome some evident limitations of previous approaches like the theory behind the so-called banana-shape regions or exact analytical solutions of the DE in the presence of highly or totally absorbing inclusions. We also propose the ER to reformulate the problem of a totally absorbing inclusion in terms of another inclusion having a finite absorption contrast and a re-scaled volume. For instance, we have shown how this approach can indeed be used to simulate black inclusions with the Born approximation. By means of comparisons with the results of Monte Carlo simulations, we have shown that the EOPTER solver can model totally absorbing inclusions with an error smaller than about $10 \%$, whereas the EOPT solver shows an error smaller than about $20 \%$, showing a performance largely better than that observed with solvers proposed previously. () 2014 Optical Society of America

OCIS codes: (170.3660) Light propagation in tissues; (170.5280) Photon migration; (170.3880) Medical and

biological imaging; (290.7050) Turbid media; (300.1030) Absorption; (290.1990) Diffusion.

http://dx.doi.org/10.1364/JOSAA.31.000460
\end{abstract}

\section{INTRODUCTION}

With this paper, we want to address the issue of photon migration in the presence of highly and totally absorbing inclusions embedded in diffusive media. The work done can be divided into two parts: a review of the literature and the proposal of suitable forward solvers for modeling of the problem investigated. The theoretical results presented in this work belong to the general framework of optical imaging through highly diffusive media (e.g., biological tissues) that is quite attractive in view of noninvasive clinical diagnostics for applications like optical mammography, functional imaging and clinical monitoring of the brain, detection of prostate tumors, assessment of ostearticular diseases, monitoring neadjuvant therapy, or photodynamic therapy, just to quote the most relevant ones [1].

Within this framework, the availability of a forward model to describe the effect of a totally absorbing (black) inhomogeneity embedded within a diffusive medium is quite relevant in four specific aspects. First, various clinical applications could present situations where optical inhomogeneity is far from being a small perturbation of the background properties, like for instance, in the case of highly perfused breast tumors, brain hemorrhages [2-4], local vessels [5], etc. A second aspect is related to the physical information that can be extracted from the perfectly absorbing case, such as the probability that a photon detected has passed through a certain region of the medium [6,7] so as to derive the density of photon paths (banana-shape regions). A third aspect is linked to the need to simulate photon propagation in phantoms with embedded black inclusions. Recently, we have demonstrated that realistic absorption perturbations can be mimicked accurately by a small black object of a proper volume []. This led to the construction of a kit of identical inhomogeneous phantoms based on black polyvinyl dichloride cylinders immersed in Intralipid and ink dilutions [8]. These kits were adopted in the nEUROPt Protocol, an interlaboratory assessment test aimed at quantifying the performance of optical brain imagers, and already applied to seven different instruments [9]. A final fourth aspect is related to the proposal to grade clinical measurements (e.g., a functional brain activation or a breast 
lesion) using equivalent black volume as a measure of the perturbation strength [8]. As a consequence, there is the need for a fast forward solver to be used either in an iterative fitting procedure or to build a look-up library for the particular geometry under study.

Several forward solvers have been proposed for describing the effects of absorbing perturbations $[6,7,10-14]$ and some of them have also been used when the absorption coefficient becomes extremely high $[6,7,10,14]$. A first application of these solvers has been the derivation of the so-called banana-shape regions in which the photon migration paths are concentrated [6,7]. These models were based on the DE that was solved by imposing a null boundary condition for the fluence on the surface of the black inclusion [6,7]. The procedure proposed in [6] and [7] provided an "exact" expression for the "banana-shape regions" (within the validity of the DE) only for a limiting case of an infinitesimal black sphere. In particular, expressions of the "bananashape regions" were provided in the infinite and semi-infinite medium geometry for the CW case. In [10], the DE was solved in the infinite medium geometry in frequency domain for a spherical defect having arbitrary size and absorption contrast with respect to the background. It is important to stress that even though the DE can be solved exactly in the region outside a black defect, to the best of our knowledge, it has never been validated in absolute terms with more rigorous solutions provided within the radiative transfer equation (RTE). Moreover, in those regions where absorption is much higher than scattering, the diffusion approximation is not expected to hold. For this evident argument, it would be desired to provide improved solutions that are able to overcome the limitations of the existing ones. Our work is also a contribution to filling this gap. In this work, we show the limitations of the DE to describe photon migration in the presence of black defects and we also propose a heuristic solver that can overcome these limitations.

Our approach is based on the formulated equivalence relation (ER) between realistic and totally absorbing optical inhomogeneities [8]. The ER states the equality between the perturbation produced by a given absorption inhomogeneity embedded in a diffusive medium and an equivalent black object of proper volume [8]. Generally speaking, for a given reduced scattering coefficient $\mu_{s}^{\prime}$ of the background medium, the ER identifies - within the set of all possible absorption inhomogeneities-subclasses of equivalent objects producing the same effect. Each subclass contains only one totally absorbing spherical inclusion, but an infinite variety of absorption inhomogeneities with different combinations of the absorption variation $\Delta \mu_{a}$ between inclusion and background, volume, and shape of the inclusion. Apart from a few extreme cases [8] -as for very low absorption perturbations quite close to a source or a detector - the relation is general; holds true for both time-resolved and CW approaches; and is fairly independent of the geometry, source-detector distance, and the location of the inhomogeneity, as well as the background absorption coefficient $\left(\mu_{a}\right)$.

In Section 2, we critically review the basic models that have been proposed to deal with the problem of totally absorbing perturbations, and present our approach based on higher order perturbative expansion and the application of the ER introduced in [8]. Practically, we propose two forward solvers: a first one simply based on eighth-order perturbation theory (EOPT) and a second one that combines EOPT with the ER (EOPTER). Moreover, we propose the application of the ER to any model-e.g., the Born approximation-as a way to reformulate the forward problem of a totally or high-absorbing inclusion in terms of another one having a milder absorption and a re-scaled volume. In Section 3, the three approaches will be tested against Monte Carlo (MC) simulations, both for the time-domain and the $\mathrm{CW}$ case, providing an overview of the performance of the solvers. In Section 4, we provide a discussion and conclusions on the work done.

\section{THEORY}

In this section, the main frame of the theories used to obtain the results presented is summarized. First, we review the theory of the banana-shape regions [6,7] and discuss how it can be generalized to any geometry. Following an approach similar to the banana-shape regions theory [6,7], we also provide an exact $\mathrm{CW}$ solution of the $\mathrm{DE}$ in the semi-infinite geometry for a non-absorbing medium, which incorporates a black spherical defect of arbitrary size. The reason for using a non-absorbing medium is that in the $\mathrm{CW}$, the DE simplifies into the Poisson equation and the method of images can be used to solve the DE exactly. Second, we describe the forward solvers presented in this paper.

\section{A. Comparisons of Standard and Existing Theories}

In the literature, we have several works dedicated to the case of a totally absorbing inclusion embedded inside a diffusive medium. All the theories mentioned in this section are based on the DE. What we want to stress is that the analytical solutions of the DE show evident lacks in their validity when describing photon migration close to totally absorbing inclusions since high values of absorption destroy the validity of the two simplifying assumptions of the diffusion approximation (see, for instance, in [15], Eq. (3.25) for the first assumption and Eq. (3.26) for the second assumption). In particular, close to totally absorbing boundaries, the diffuse intensity does not have an almost isotropic angular distribution and cannot be approximated by the first two terms of a series expansion in spherical harmonics. Moreover, close to black boundaries, due to the steep spatial and angular variations of the specific intensity, large values of the photon flux and of its derivative are also expected, and the second assumption of the diffusion approximation is likely to fail [15]. Therefore, the accuracy of solvers based on the DE should be questioned and compared with the exact solution of the RTE even when the other parameters of the geometry (like source-detector distance) and the optical properties outside the black defect are within the range of validity of the DE.

We start this section with the simulation of the effect of a totally absorbing inhomogeneity for the $\mathrm{CW}$ case using three already available models, which constitutes the state of the art in the field. This comparison provides the starting point and the motivation to this work. The three models-including some original derivation-will be presented in detail afterward, together with an insight into the assumptions and implications. In Fig. 1, we show a comparison of the results of MC simulations and of other three existing theories for a semi-infinite medium with a black sphere located at depth $z=15 \mathrm{~mm}$, while its center varies in the range 


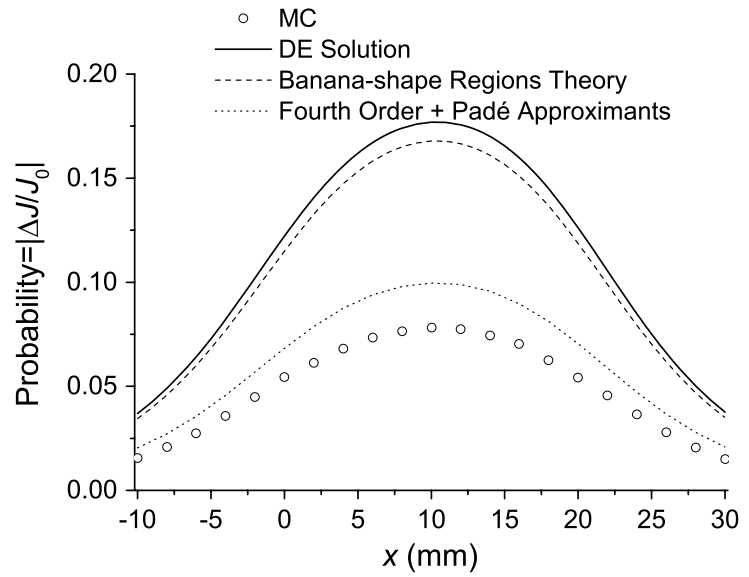

Fig. 1. Comparison of the probability of a photon detected to "pass" through or "intersect" the region occupied by a black sphere of radius $2 \mathrm{~mm}$ placed inside a semi-infinite medium obtained with various solvers.

$x \in(-10,30) \mathrm{mm}$ and $y=0(x, y$ Cartesian coordinates on the external plane of the medium at $z=0$ ). The probability of a photon detected to "pass" through or "intersect" the region occupied by the black sphere is plotted versus the scanning coordinate $(x)$. This probability is calculated as the absolute value of the relative change of the detected CW flux, $\left|\Delta J / J_{0}\right|$, with respect to its baseline value $J_{0}$ (i.e., the value without the defect). Figure 1 pertains to a medium having $\mu_{s}^{\prime}=0.5 \mathrm{~mm}^{-1}$ and $\mu_{a}=0$, and the refractive indices inside and outside the medium are 1.4 and 1, respectively. An isotropic source placed at $\left(0,0,1 / \mu_{s}^{\prime}\right)$ has been considered for all the solvers. The detector is located at $(20,0,0) \mathrm{mm}$. For the MC simulation, the detector is a square of side $2 \mathrm{~mm}$ and the simulation was run for isotropic scattering and terminated after detecting $10^{4}$ photons. The fourth-order perturbation theory-based results are obtained with $\Delta \mu_{a}=6 \mathrm{~mm}^{-1}$, which practically simulates a totally absorbing inclusion. The curve labeled "DE Solution" is the exact solution of the DE obtained with the method of images (so far, we know this solution is unpublished) and was obtained with 40 image point sources. The other two curves were obtained with the theory of the banana regions [6,7] and with a heuristic fourth-order perturbation theory integrated with the method of Padé approximants [16-19]. The results clearly show the deficiencies of the theories based on rigorous solutions of the DE when used in the presence of totally absorbing inclusions. A better agreement with MC simulations is obtained with the heuristic theory developed by Sassaroli and co-workers [16-19]. In what follows, we introduce the theories used in Fig. 1 .

\section{Banana-Shape Regions Theory}

In this section, we describe the main frame of the bananashape regions theory [6,7]. We derive analytical formulas for the relative $\mathrm{CW}$ perturbation that slightly differ (by a normalizing factor) from those obtained in [] $]$ and [7]], although the approach followed is basically the same. The exact meaning of using a "small" totally absorbing object to "probe" a diffusive light field is to provide the probability of a photon detected to intersect (or pass through) the region where the object is located. For simplicity, we can consider the infinite medium geometry; however, later we will state that the "banana-shape" regions approach can also be generalized to an arbitrary bounded geometry (not only semi-infinite as the authors considered in their paper). The method of the banana-shape regions consists of matching the boundary conditions at the exterior of the black sphere (i.e., on its surface), that is, $\Phi\left(\mathbf{r}_{s}, \mathbf{r}\right)=0$ for any $\mathbf{r}$ at the boundary of the sphere (i.e., the total fluence at the surface of the sphere is " 0 "). In the argument of $\Phi$, the first point is always the source point and the second is the field point, with $\mathbf{r}_{\mathbf{s}}$ position vector of the real source that is also the origin of the reference system so that $\mathbf{r}_{s}=0$. In order to do so, we consider a negative point source (abbreviated as ns as opposed to rs, which means "real source") at the center of the sphere so that $\Phi\left(\mathbf{r}_{s}, \mathbf{r}_{1}+\mathbf{r}_{a}\right)=\Phi_{r s}\left(\mathbf{r}_{s}, \mathbf{r}_{1}+\mathbf{r}_{a}\right)+\Phi_{n s}\left(\mathbf{r}_{1}, \mathbf{r}_{a}\right)$ (r. $\mathbf{r}_{1}$ position vector from the real source to the center of the totally absorbing sphere; $\mathbf{r}_{a}$ position vector from the center of the sphere to its external; $\mathbf{r}_{1}+\mathbf{r}_{a}$ position vector from the real source to the external of the sphere). If $\left|\mathbf{r}_{1}\right| \gg\left|\mathbf{r}_{a}\right|$ (which we rewrite as $r_{1} \gg r_{a}=a$; we follow the convention that a symbol indicating a position if not a vector represents its modulus), we can write $\Phi\left(\mathbf{r}_{s}, \mathbf{r}_{1}+\mathbf{r}_{a}\right) \cong \Phi_{r s}\left(\mathbf{r}_{s}, \mathbf{r}_{1}\right)+\Phi_{n s}\left(\mathbf{r}_{1}, \mathbf{r}_{a}\right)=0$. Explicitly, we have this condition for the total fluence:

$$
\frac{S_{0}}{4 \pi D} \frac{\exp \left(-\mu_{\mathrm{eff}} r_{1}\right)}{r_{1}}+\frac{q}{4 \pi D} \frac{\exp \left(-\mu_{\mathrm{eff}} a\right)}{a}=0
$$

where $S_{0}$ is the strength of the real source, $\mu_{\text {eff }}=\sqrt{3 \mu_{a} \mu_{s}^{\prime}}$ is the effective attenuation coefficient, and $D=1 /\left(3 \mu_{s}^{\prime}\right)$ is the diffusion coefficient [15]. From Eq. (1), we can derive the source "strength" $q$ (which will be negative and will have the same units as of $S_{0}$, i.e., number of photons/second). Once $q$ has been derived, we can obtain the relative perturbation at the detector's site $\mathbf{r}_{d}$ as

$$
\frac{\Delta \Phi}{\Phi_{0}}\left(\mathbf{r}_{s}, \mathbf{r}_{d}\right)=-\frac{\frac{a}{r_{1}} \exp \left[-\mu_{\mathrm{eff}}\left(r_{1}-a\right)\right] \frac{\exp \left[-\mu_{\mathrm{eff}}\left|\mathbf{r}_{d}-\mathbf{r}_{1}\right|\right]}{\left|\mathbf{r}_{d}-\mathbf{r}_{1}\right|}}{\frac{\exp \left(-\mu_{\mathrm{eff}} r_{d}\right)}{r_{d}}} .
$$

In the original paper [6], the authors used a different definition of $q$ [which incorporated the factor $4 \pi D$ in Eq. (1)]; moreover, when they studied the probability of a photon detected to pass through the sphere, they considered the limiting case for $a$ approaching " 0 " of $(\Delta \Phi / a)$, leading to an expression that they called the non-normalized probability of a photon detected to intersect the black sphere. Instead, the correct way to define this probability by using their approach is to realize that Eq. (2) is already the opposite of a probability and it will approach the correct solution as $a$ approaches "0" (note that the probability approaches " 0 " as the radius $a$ approaches " 0 ," i.e., the order of infinitesimal is $a$ ):

$$
P_{\mathrm{bs}}=-\left.\frac{\Delta \Phi}{\Phi_{0}}\left(\mathbf{r}_{s}, \mathbf{r}_{d}\right)\right|_{\mathrm{bs}},
$$

where bs stands for black sphere. We note that the reasoning of Feng et al. []ㅡ, which used for the probability $(\Delta \Phi / a)$, already contains a wrong assumption, that is, the behavior of the solution of the $\mathrm{DE}$ in the infinite medium geometry when the source-detector distance approaches " 0 " [which is $\alpha(1 / r)$ ] correctly represents the fluence in the vicinity of the source. Instead, we know from the improved solution provided by Graaf and Rinzema [20] that the solution when $r$ approaches " 0 " behaves as $\alpha\left(1 / r^{2}\right)$. This is exactly in agreement with the RTE as when the source-detector 
distance $r$ approaches " 0 ," the contribution to the fluence is mainly made by the ballistic component, that is, $\Phi_{b}=\exp \left[-\left(\mu_{a}+\mu_{s}\right) r\right] /\left(4 \pi r^{2}\right)$, with $\mu_{s}$ scattering coefficient, whereas the diffuse component of the fluence tends to " 0. ." It should be noted immediately that the contribution due to the ballistic component when $r$ approaches "0" behaves as $\alpha\left(1 / r^{2}\right)$. Therefore, if one repeats all the steps, then the limit in Eq. (3) when $a$ approaches " 0 " is an infinitesimal of the order of $a^{2}$. Nevertheless, we will keep the assumptions suggested by the authors, to be consistent with their method and to develop its consequences in a bounded geometry.

We conclude this brief review on the banana-shape regions theory by noting the link between this theory and the Born approximation. If we are using the first-order perturbation theory (Born approximation), we cannot deal with a black sphere, but only with "small" contrast perturbations $\left(\Delta \mu_{a}\right)$. The expression that can be derived within this theory is [16]

$$
\left.\frac{\Delta \Phi}{\Phi_{0}}\left(\mathbf{r}_{s}, \mathbf{r}_{d}\right)\right|_{\text {Born }} \approx-\left\langle l_{i}\right\rangle \Delta \mu_{a}
$$

where $\left\langle l_{i}\right\rangle$ is the mean path length and can be expressed for small volumes far enough from the source and the receiver as $[16]$

$$
\left\langle l_{i}\right\rangle=\frac{\int_{V} \Phi_{0}\left(\mathbf{r}_{s}, \mathbf{r}^{\prime}\right) \Phi_{0}\left(\mathbf{r}^{\prime}, \mathbf{r}_{d}\right) \mathrm{d} V^{\prime}}{\Phi_{0}\left(\mathbf{r}_{s}, \mathbf{r}_{d}\right)} \cong \frac{\Phi_{0}\left(\mathbf{r}_{s}, \mathbf{r}_{1}\right) \Phi_{0}\left(\mathbf{r}_{1}, \mathbf{r}_{d}\right)}{\Phi_{0}\left(\mathbf{r}_{s}, \mathbf{r}_{d}\right)} V,
$$

where $V$ is the volume of the region occupied by the black sphere $\left[V=(4 / 3) \pi a^{3}\right]$. Therefore, Eq. ( $\left.\underline{4}\right)$ can be rewritten as

$$
\left.\frac{\Delta \Phi}{\Phi_{0}}\left(\mathbf{r}_{s}, \mathbf{r}_{d}\right)\right|_{\text {Born }}=-\frac{\frac{a^{3}}{3 D r_{1}} \exp \left[-\mu_{\mathrm{eff}} r_{1}\right] \frac{\exp \left[-\mu_{\mathrm{eff}}\left|\mathbf{r}_{d}-\mathbf{r}_{1}\right|\right]}{\left|\mathbf{r}_{d}-\mathbf{r}_{1}\right|}}{\frac{\exp \left(-\mu_{\mathrm{eff}} r_{d}\right)}{r_{d}}} \Delta \mu_{a} .
$$

We can see a similarity between Eqs. (6) and (2) [even though the order of infinitesimal in Eq. (6) is $a^{3}$ as opposed to $a$ in Eq. (2)]. More precisely, we have

$$
\left.\frac{\Delta \Phi}{\Phi_{0}}\left(\mathbf{r}_{s}, \mathbf{r}_{d}\right)\right|_{\mathrm{bs}}=\left.\frac{\Delta \Phi}{\Phi_{0}}\left(\mathbf{r}_{s}, \mathbf{r}_{d}\right)\right|_{\text {Born }} \frac{3 D}{a^{2}} \exp \left(\mu_{\mathrm{eff}} a\right) \frac{1}{\Delta \mu_{a}}
$$

We also note that $P_{\mathrm{bs}}$ can be expressed in terms of $\left\langle l_{i}\right\rangle$ :

$$
P_{\mathrm{bs}}=-\left.\frac{\Delta \Phi}{\Phi_{0}}\left(\mathbf{r}_{s}, \mathbf{r}_{d}\right)\right|_{\mathrm{bs}}=\left\langle l_{i}\right\rangle \frac{3 D}{a^{2}} \exp \left(\mu_{\mathrm{eff}} a\right)=\frac{\left\langle l_{i}\right\rangle}{V \Phi_{0}\left(r_{1}, a\right)}
$$

In Eq. (8), $\Phi_{0}\left(r_{1}, a\right)$ is the fluence calculated at the surface of the sphere due to a point source of unitary strength at its center. In substance, the banana-shape regions approach is a mean path length approach. This conclusion follows also from the observation of this relationship:

$$
\begin{aligned}
\lim _{V \rightarrow 0} \frac{\left\langle l_{i}\right\rangle}{V} & =-\frac{\frac{1}{3 D r_{1}} \exp \left[-\mu_{\mathrm{eff}} r_{1}\right] \frac{\left.\exp \left[-\mu_{\mathrm{eff}} \mid \mathbf{r}_{d}-\mathbf{r}_{1}\right]\right]}{\left|\mathbf{r}_{d}-\mathbf{r}_{1}\right|}}{\frac{\exp \left(-\mu_{\mathrm{eff}} r_{d}\right)}{r_{d}}} \\
& =-\left.\frac{1}{4 \pi D} \lim _{a \rightarrow 0} \frac{1}{a} \frac{\Delta \Phi}{\Phi_{0}}\left(\mathbf{r}_{s}, \mathbf{r}_{d}\right)\right|_{\mathrm{bs}}
\end{aligned}
$$

Without retracing the previous steps, we state that Eq. (8) can be used to calculate the probability of a photon detected to intersect a certain spherical region (i.e., to be absorbed by a black sphere located in that region) in any kind of bounded geometry. The proof will be straightforward if one repeats all the steps. Finally, we note that the similarity found between the banana-shape expression and the one obtained with the Born approximation is a direct consequence of the ER [].

\section{Exact Solution of the $D E$}

While the banana-shape regions theory can be developed in general for any bounded geometry and for any value of the absorption coefficient of the medium (within the validity of the DE), it yields the correct perturbation only for the limiting case of a "small" spherical defect (i.e., for a defect having a linear size much smaller than the distance between its center and the source). Here, we are presenting the CW solution of the DE for a non-absorbing semi-infinite medium, which does not have this restriction and therefore, it is valid for any size of the black sphere embedded in the medium. For a CW source and a non-absorbing medium, the DE is

$$
D \nabla^{2} \Phi(\mathbf{r})=\delta\left(\mathbf{r}-\mathbf{r}_{s}\right) .
$$

Equation (10) is valid in any field point outside the sphere. In the infinite medium geometry, if we choose a point source located in a particular position inside the sphere and having a particular strength, the boundary condition of the fluence ( $\Phi=0$ in all the points of the surface) can be met. By using the standard solution derived in electrostatic for the potential generated by a point charge in front of a spherical conductor at null potential [21], we can prove that the solution is obtained by choosing a source strength equal to $a / R$ of the source strength of the real source (in this case, " 1 "), where $a$ is the radius of the sphere and $R$ the distance between the real source and the center of the sphere. Also, the location of the image point source inside the sphere is at distance $b=a^{2} / R$ from its center. Therefore, outside the sphere, the solution of the DE is

$$
\Phi(\mathbf{r})=\frac{1}{4 \pi D}\left[\frac{1}{\left|\mathbf{r}-\mathbf{r}_{s}\right|}-(a / R) \frac{1}{\left|\mathbf{r}-\left(a^{2} / R\right) \hat{k}\right|}\right] .
$$

In Eq. (11), $\mathbf{r}$ is the location of an arbitrary field point and the $z$ axis is chosen to connect the center of the sphere and the point source, and $\hat{k}$ is the unit vector of $z$ axis. Note that from Eq. (11) and from the expression of the fluence for the homogeneous medium, we can derive $\Delta \Phi / \Phi_{0}$, which in the limit for $a$ approaching " 0 " is coincident with Eq. (2) obtained with the banana-shape regions theory. This result reinforces the statement that both the theories considered are based on the fundamental parameter $\left\langle l_{i}\right\rangle$ used in the first-order perturbation theory, and also explains the results of Fig. 1 where the curves obtained with the banana-shape regions theory and the DE solution are very close to each other.

Now let us consider how to build the solution for a black sphere in a non-absorbing semi-infinite medium using the method of images. For handling correctly the boundary conditions at both the surface of the black sphere and at the boundary of the semi-infinite medium, we need an infinite series of image sources inside the sphere and also an infinite series of mirror images in the semi-space not occupied by the diffusive medium. Inside the sphere, the series of image 


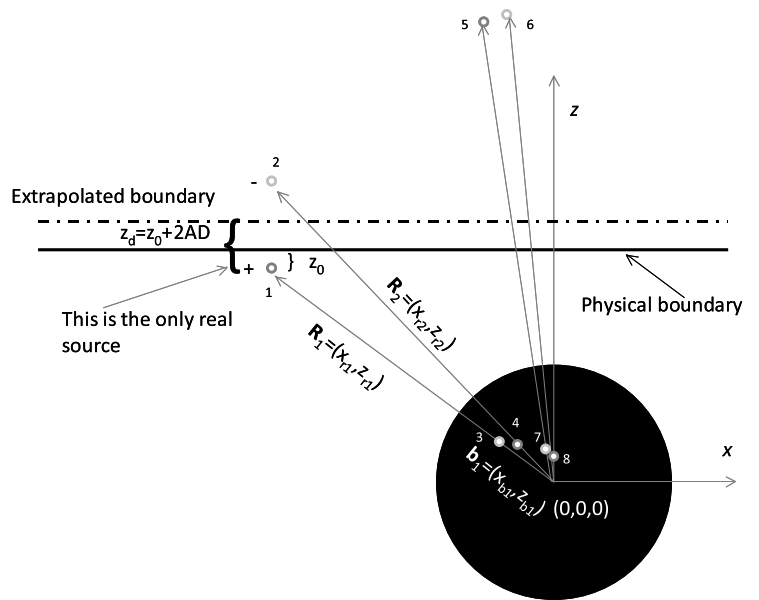

Fig. 2. Mirror image sources inside the semi-infinite medium.

sources will get closer to the center of the sphere (and their strength smaller) as we increase the order of the approximation. The positions of the image sources outside the sphere are indicated by vectors $\mathbf{R}_{i}=\left(x_{r i}, z_{r i}\right)$; also, the position of the image sources inside the sphere are indicated by vectors $\mathbf{b}_{i}=\left(x_{b i}, z_{b i}\right)$. Note that $R_{i}=\left|\mathbf{R}_{i}\right|$ and $b_{i}=\left|\mathbf{b}_{i}\right|$ (see Fig. $\underline{2}$ and Table 1). The directions of the image sources can be $\bar{i}$ ndicated by using the unit vectors $\hat{u}_{r i}$ and $\hat{u}_{b i}$. We note that the only real source in the table is the first one and that from the knowledge of its position, we can derive the positions and the strengths of all the other image sources.

\section{Fourth-Order Heuristic Theory Based on the DE}

About the fourth-order heuristic theory proposed by Sassaroli and co-workers [ $\underline{16}-\underline{19}]$, we only remind that it originated from a solution of the higher order perturbation theory of the DE, for which the higher order path length moments were derived by using MC simulations in a wide range of geometries and optical properties of interest in near-infrared spectroscopy (NIRS) and diffuse optical tomography (DOT). The comparison in Fig. 1 shows that although there are still discrepancies with the results of MC simulations, this solution is significantly better than the solutions purely based on the DE. In the next section, while introducing the forward

Table 1. Positions of the Image Sources of the DE Solution for a Semi-infinite Medium

\begin{tabular}{lc}
$x$ & $z$ \\
$x_{r 1}$ & $z_{r 1}$ \\
$x_{r 2}=x_{r 1}$ & $z_{r 2}=z_{r 1}+2 z_{d}$ \\
$x_{r 2 k+1}=x_{b 2 k-1}$ & $z_{r 2 k+1}=z_{r 2}+z_{r 1}-z_{b 2 k-1}$ \\
$x_{r 2 k+2}=x_{b 2 k}$ & $z_{r 2 k+2}=z_{r 2}+z_{r 1}-z_{b 2 k}$ \\
$x_{b 2 k-1}=\left(a^{2} / R_{2 k-1}\right) u_{r 2 k-1}$ & $z_{b 2 k-1}=\left(a^{2} / R_{2 k-1}\right) u_{r 2 k-1 z}$ \\
$x_{b 2 k}=\left(a^{2} / R_{2 k}\right) u_{r 2 k x}$ & $z_{b 2 k}=\left(a^{2} / R_{2 k}\right) u_{r 2 k z}$ \\
$\hat{u}($ unit vector $)$ & Strength \\
$\hat{u}_{r 1}=\left(x_{r 1}, z_{r 1}\right) / R_{1}$ & $S=1$ \\
$\hat{u}_{r 2}=\left(x_{r 2}, z_{r 2}\right) / R_{2}$ & $S=-1$ \\
$\hat{u}_{r 2 k+1}=\left(x_{r 2 k+1}, z_{r 2 k+1}\right) / R_{2 k+1}$ & $S=\prod_{l>0 ; 2 k-l>0}^{(\text {lodd })}\left(a / R_{2 k-l}\right)$ \\
$\hat{u}_{r 2 k+2}=\left(x_{r 2 k+2}, z_{r 2 k+2}\right) / R_{2 k+2}$ & $S=-\prod_{l \geq 0 ; 2 k-l>0}^{(\text {leven })}\left(a / R_{2 k-l}\right)$ \\
$\hat{u}_{b 2 k-1}=\hat{u}_{r 2 k-1}$ & $S=-\prod_{l>0 ; 2 k-l>0}^{\text {lodd) }}\left(a / R_{2 k-l}\right)$ \\
$\hat{u}_{b 2 k}=\hat{u}_{r 2 k}$ & $S=\prod_{l \geq 0 ; 2 k-l>0}^{\text {(leven })}\left(a / R_{2 k-l}\right)$ \\
\hline
\end{tabular}

solver proposed in this paper, we will also retrace the basic concepts of the higher order theory.

\section{B. Improved Forward Solvers}

In this section, we propose to calculate the effects of totally absorbing objects embedded inside diffusive media by means of a high-order perturbation theory [16-19] and the ER described in [8]. We have started our derivation from the solver proposed by Sassaroli and co-workers [16-19], a fourth-order perturbation theory by use of the moments of the generalized temporal point-spread function that was combined together with the method of Padé approximants. The main lack of a high-order perturbation theory when dealing with black defects is the convergence of the series representing the solution. The general solution presented by Sassaroli and co-workers [ $\underline{16}-19]$ is practically a Taylor expansion in terms of the absorption variation $\Delta \mu_{a}$ between an inclusion and the background medium. The description of a black defect can be obtained by inserting for $\Delta \mu_{a}$ a value sufficiently high, but at the same time, the number of terms required in the Taylor expansion increases as long as $\Delta \mu_{a}$ increases. In order to improve this theory, we have acted in two directions: we have doubled the number of terms of the perturbation theory passing to an EOPT and, by using the ER, we have accounted for the effect of the black defect by replacing it with its equivalent volume with a significantly lower value of $\Delta \mu_{a}$. These two changes produce a significant improvement in terms of the convergence of the proposed solver compared with the previous one. In this section, we summarize the main frame of the EOPT and recall the ER used to replace a totally absorbing defect with an equivalent realistic absorbing volume.

\section{Eighth-Order Perturbation Theory}

In accordance with the theory described in [16-19], given a single defect of volume $V$ placed inside a diffusive medium at $\mathbf{r}_{i}$, a point source of unitary strength located at $\mathbf{r}_{s}$ inside the medium, represented by the function $\delta\left(\mathbf{r}-\mathbf{r}_{s}\right) \delta(t)$ for the time domain and the function $\delta\left(\mathbf{r}-\mathbf{r}_{s}\right)$ for the CW domain, and a point of collection $\mathbf{r}_{b}$ located at the boundary of the medium, indifferently for the time or CW domain, the eighth-order contrast for the flux exiting a diffusive medium and for a single defect can be written, limiting the series to the first eight terms, as [16-19]

$$
C\left(\mathbf{r}_{s}, \mathbf{r}_{b}, V, \Delta \mu_{a}\right)=\frac{J-J_{0}}{J_{0}} \approx \sum_{n=1}^{8} \frac{(-1)^{n}}{n !}\left\langle l_{i}^{n}\right\rangle\left(\Delta \mu_{a}\right)^{n},
$$

with $J_{0}$ the photon flux of the background medium (initial state without inclusion $V$ ), $J$ the photon flux of the perturbed medium (final state with inclusion $V$ ), $\left\langle l_{i}^{n}\right\rangle$ the mean path length self-moments related to volume $V$, and $\Delta \mu_{a}$ the absorption variation of volume $V$ with respect to the background (initial state). We would like to stress that the mean path length self-moments in the time and $\mathrm{CW}$ domains assume different values in accordance with their proper definition, so that they cannot be used interchangeably with the same meaning in the two domains. For the time domain, we have that both $\left\langle l_{i}^{n}\right\rangle$ and contrast depend on time. The coefficients $\left\langle l_{i}^{n}\right\rangle\left(\mathbf{r}_{b}\right)$ for the CW domain and the coefficients $\left\langle l_{i}^{n}\right\rangle\left(\mathbf{r}_{b}, t\right)$ for the time domain are the key quantities of Eq. (12) and for their calculation, we apply the following relations [16-19]: 


$$
\begin{aligned}
\left\langle l_{i}^{n}\right\rangle\left(\mathbf{r}_{b}\right) \approx & k_{n-1}\left\langle l_{i}\right\rangle\left(\mathbf{r}_{b}\right)\left(\int_{V} \Phi_{0}\left(\mathbf{r}_{\mathbf{i}}, \mathbf{r}\right) \mathrm{d} \mathbf{r}\right)^{n-1} \\
\left\langle l_{i}^{n}\right\rangle\left(\mathbf{r}_{b}, t\right) \approx & \frac{k_{n-1}}{J_{0}\left(\mathbf{r}_{b}, t\right)}\left\{\left[J_{0}\left(\mathbf{r}_{b}, \tau\right)\left\langle l_{i}\right\rangle\left(\mathbf{r}_{b}, \tau\right)\right]\right. \\
& \left.\otimes{ }_{j=1}^{n-1} \int_{V} \Phi_{0}\left(\mathbf{r}_{\mathbf{i}}, \mathbf{r}, \tau\right) \mathrm{d} \mathbf{r}\right\}(t) \quad n \geq 2,
\end{aligned}
$$

with $V$ the volume of the defect, $\Phi_{0}$ and $J_{0}$ the photon fluence and the photon flux rate in the initial state, $\otimes$ the convolution integral symbol, $\mathbf{r}_{b}$ the collection point at the boundary of the medium, and $\mathbf{r}_{i}$ the position vector of the defect (for spherical regions, we choose $\mathbf{r}_{i}$ at their centers); coefficients $k_{n-1}$ are reported in Table 2 . In the previous fourth-order theory [17-19], we reported the coefficient up to $k_{3}$, whereas here we provide the coefficients up to $k_{7}$ so that eighth-order calculations can be carried out. These coefficients have been calculated with MC simulations that have been repeated for several combinations of optical properties, position, and size of the inclusion, and geometry. For more details on the statistical study needed to determine average values of these coefficients, see Ref. [17]. Only slight differences have been observed in the coefficients by changing the refractive index mismatch $n$ between the medium and the external region in the range 1-1.4. The errors affecting the determination of these coefficients are also affecting the accuracy of the overall forward model. We would like to stress that these coefficients are the same for the time and CW domains. Therefore, from the prior calculation of the mean path length $\left\langle l_{i}\right\rangle$ spent inside volume $V$, it is possible to calculate all self-moments up to the eighth order. The value of $\left\langle l_{i}\right\rangle$ is calculated by using the standard diffusion theory making use of the Born approximation. We have already shown elsewhere that the DE provides with good accuracy the information on mean path length [12]. Finally, evaluation of Eq. (13) also requires the calculation of the integral term of the fluence rate on volume $V$. For evaluation of this integral, we have used, for the CW case, the improved DE solution for the infinite medium proposed in [20] and, for the time domain, the solution of the RTE for the infinite medium geometry and isotropic scattering proposed by Paasschens [22]. The calculation of contrast $C$ is finalized by using the method of Padé approximants so that the lefthand side of the Taylor expansion in Eq. (12) is approximated with the rational function

$$
C \approx P_{M, N}\left(\Delta \mu_{a}\right)=\sum_{k=0}^{M} a_{k}\left(\Delta \mu_{a}\right)^{k} /\left[1+\sum_{j=0}^{N} b_{j}\left(\Delta \mu_{a}\right)^{j}\right] .
$$

The method of Padé approximants is used to extend the validity of the theory to a wider range of absorption contrasts between defects and background. For our results, we used $M=N=4$ because only nine coefficients can be derived from Eq. (12) (the first being 0). Coefficients $a_{k}$ and $b_{j}$ are calculated by imposing that the values of $P_{M, N}\left(\Delta \mu_{a}\right)$ and its first
$M+N$ derivatives calculated at $\Delta \mu_{a}=0$ coincide with those of Eq. (12): for the time domain, this condition has be imposed at each time $t$ and for the CW case, at each source-detector distance.

\section{Equivalence Relation}

The second part of the forward calculation proposed in this paper involves the use of the ER so that a small and completely black object can give almost the same relative perturbation as a larger object with only moderate absorption. We have provided (see Eq. (8) in [8]) some interpolating functions that, given a black object of volume $V_{\text {black }}$ located in a diffusive medium with reduced scattering coefficient $\mu_{s}^{\prime}$, provide the volume $V$ and the absorption variation $\Delta \mu_{a}$ of the equivalent realistic inhomogeneity. With the values of $\Delta \mu_{a}$ and $V$ and the eighth-order theory described above [Eqs. (12) and (14)], it is therefore possible to evaluate the contrast produced by the black defect under investigation. The benefit of the ER in the calculation of the relative contrast is related to the fact that using the equivalent volume the truncated series of Eq. (12) is exploited for smaller values of $\Delta \mu_{a}$ for which better convergence properties can be obtained.

\section{Forward Solvers and Methodology}

The EOPT [contrast obtained with Eqs. (12) and (14)] provides a forward solver that can be used for both highly or totally absorbing inclusions. Due to the saturation effect of an absorbing perturbation, the case of a totally absorbing inclusion can be practically simulated with an inclusion with a high finite contrast $\Delta \mu_{a}$. For many real cases, an inclusion with $\Delta \mu_{a}>5 \mathrm{~mm}^{-1}$ has an effect almost equivalent to that of a totally absorbing object. We would like to stress that in general, the saturation value for $\Delta \mu_{a}$ depends on the volume of the inclusion and decreases for larger volumes. The combination of the EOPT solver with the ER returns a second forward solver that we denote by the acronym eighth-order perturbation theory and equivalence relation (EOPTER). This solver is specially dedicated to totally absorbing objects that, thanks to the equivalent relation, are calculated by using the equivalent realistic volume $V$ (we have used $V=1000 \mathrm{~mm}^{3}$ in accordance to our previous paper [8]) with the related realistic absorption variation $\Delta \mu_{a}$ obtained from the interpolating formulae presented in [8].

Finally, we would like to stress that the ER can be combined with any kind of forward solver for calculation of totally absorbing inclusions. As an example, we consider the wellknown Born approximation combined with the ER. This solver will be denoted as Born approximation and equivalence relation (BAER).

At last, we list the main sources of approximation of the proposed forward solvers. First, we mention the intrinsic approximations related to the truncated series of Eq. (12) and the approximated values provided for $\left\langle l_{i}^{n}\right\rangle$ affecting the

\begin{tabular}{|c|c|c|c|c|c|c|}
\hline$k_{1}$ & $k_{2}$ & $k_{3}$ & $k_{4}$ & $k_{5}$ & $k_{6}$ & $k_{7}$ \\
\hline $1.50 \pm 0.03$ & $3.2 \pm 0.1$ & $9.1 \pm 0.5$ & $32 \pm 2$ & $135 \pm 12$ & $667 \pm 82$ & $3744 \pm 680$ \\
\hline
\end{tabular}
EOPT and EOPTER solvers. Second, about the proposed

Table 2. Coefficients $k_{n}$ and their Standard Deviations Calculated with MC Simulations by Average of Several Combinations of Optical Properties, Position, and Size of Inclusion, and Geometry 
methodology (used for the EOPTER and BAER solvers), we mention the intrinsic approximations of the ER [8].

\section{ANALYTICAL SOLVERS VERSUS MC SIMULATIONS}

In this section, we have used MC simulations as a gold standard to investigate the accuracy of the proposed forward solvers to describe photon migration in the presence of totally and high-absorbing inclusions. Indeed, the method detailed in this section consists of dividing the comparisons between MC simulations and analytical solvers into progressive steps. The diverse steps identify different volume ranges of the inclusion, different domains of analysis, and different solvers. A perturbation MC code was used to simulate photon migration through absorbing objects placed inside turbid media $[15,23]$ in order to have reference data for our investigations. We would like to point out that all simulated MC data presented in this section were obtained by using an isotropic scattering function.

\section{A. Time Domain}

We consider a Dirac $\delta(t)$ pencil beam source (propagating along $z$ axis) impinging normally on the medium at the entrance point [origin of the orthogonal coordinate system $(x, y, z)]$. Figure $\underline{3}$ (reflectance from a semi-infinite medium) and Fig. 4 (transmittance through a slab $4 \mathrm{~cm}$ thick) show a comparison of the relative temporal contrast $|C(t)|$ between the EOPT and EOPTER solvers with the results of MC simulations. In particular, three different totally absorbing inclusions of volumes 5,50 , and $100 \mathrm{~mm}^{3}$ set at different depths (columns) $\left(z_{i} / \mathrm{mm}=10,15,20\right.$ for reflectance and $z_{i} / \mathrm{mm}=30,25,20$ for transmittance) were considered. The
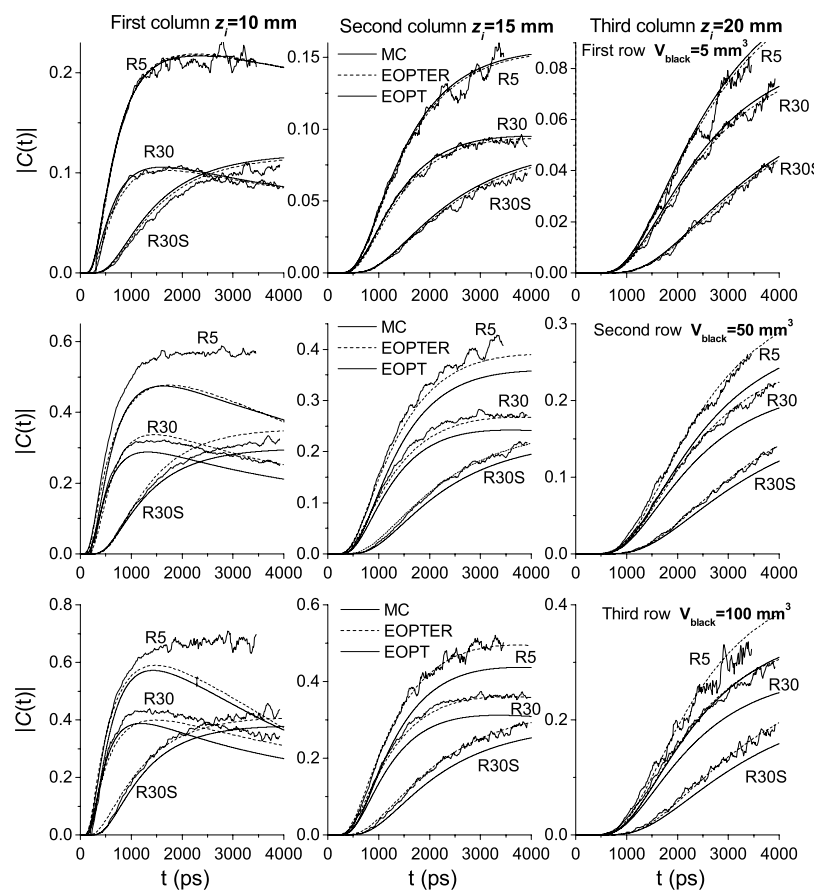

Fig. 3. Time-resolved relative contrast $|C(t)|$ for reflectance obtained with MC simulations, and with the EOPTER and the EOPT solvers for a black inclusion located inside a semi-infinite medium with $\mu_{s}^{\prime}=1 \mathrm{~mm}^{-1}$. The plots pertain to three different arrangements of detector and inclusion.
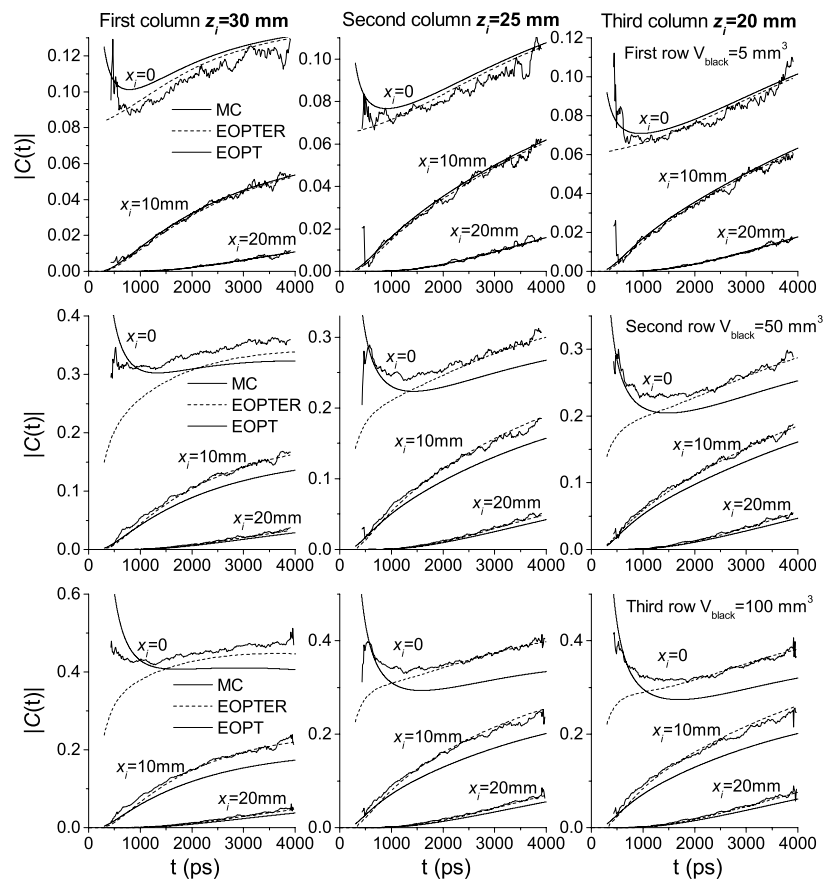

Fig. 4. Time-resolved relative contrast $|C(t)|$ for transmittance obtained with MC simulations, with the EOPTER and EOPT solvers for a black inclusion located inside a slab $40 \mathrm{~mm}$ thick slab with $\mu_{s}^{\prime}=1 \mathrm{~mm}^{-1}$. The plots pertain to three different arrangements of the inclusion [positions $\left(x_{i}, y_{i}, z_{i}\right)$ ].

refractive index of the medium and the external region was 1.33 , and $\mu_{s}^{\prime}=1 \mathrm{~mm}^{-1}$ in both figures. We would like to stress that the results are independent of $\mu_{a}$. In Fig. 3, we have reflectance measurements from a semi-infinite medium at source-detector distances $\rho=5 \mathrm{~mm}$ (R5) and $\rho=30 \mathrm{~mm}$ (R30 and R30S): the inclusion is set midway between the source [placed at $(0,0,0)$ ] and the detector [placed at $(5,0,0) \mathrm{mm}$ for R5 and at $(30,0,0) \mathrm{mm}$ for R30] for R5 and $\mathrm{R} 30\left(x_{i} / \mathrm{mm}=2.5,15, y_{i}=0\right)$, and with an $x$-offset of $15 \mathrm{~mm}$ with respect to the midpoint for R30S (that has $x_{i}=30 \mathrm{~mm}, y_{i}=0$ ). In Fig. 4 , we have transmittance measurements through a $4 \mathrm{~cm}$ thick slab (for three configurations of the inclusion) with the detector at $x=y=0$ and $z=40 \mathrm{~mm}$ : the inclusion is set on-axis for $\left(x_{i}=y_{i}=0\right)$ and off-axis with an $x$-offset of 10 and $20 \mathrm{~mm}$, respectively, that is, $x_{i}=10 \mathrm{~mm}, y_{i}=0$ and $x_{i}=20 \mathrm{~mm}, y_{i}=0$. The EOPT solver is used assuming a value of $\Delta \mu_{a}=10 \mathrm{~mm}^{-1}$, which practically simulates a totally absorbing inclusion. We note that for the overall set of comparisons, the EOPTER solver shows better agreement with the MC results than the EOPT solver. This fact confirms that the ER improves the performance of the EOPT solver. We would also like to stress that in Fig. 3, for the case with $V=100 \mathrm{~mm}^{3}$ and $z_{i}=20 \mathrm{~mm}$, the EOPT curve for R5 is very close to the EOPTER curve for R30 so that they are almost undistinguishable. In Fig. 3, the largest deviation occurs for the detector at $5 \mathrm{~mm}$ (R5) for the shallower depth $z_{i}=10 \mathrm{~mm}$. For this situation, in which the black inclusions are only a few millimeters from the source and the detector, we expect that the two simplifying assumptions of the diffusion approximations may fail. Also for Fig. $\underline{4}$, the worst comparison is obtained when the inclusion is closer to the detector, that is, for the case $x_{i}=0, z_{i}=30 \mathrm{~mm}$. Therefore, we observe that a significant reduction in accuracy is 

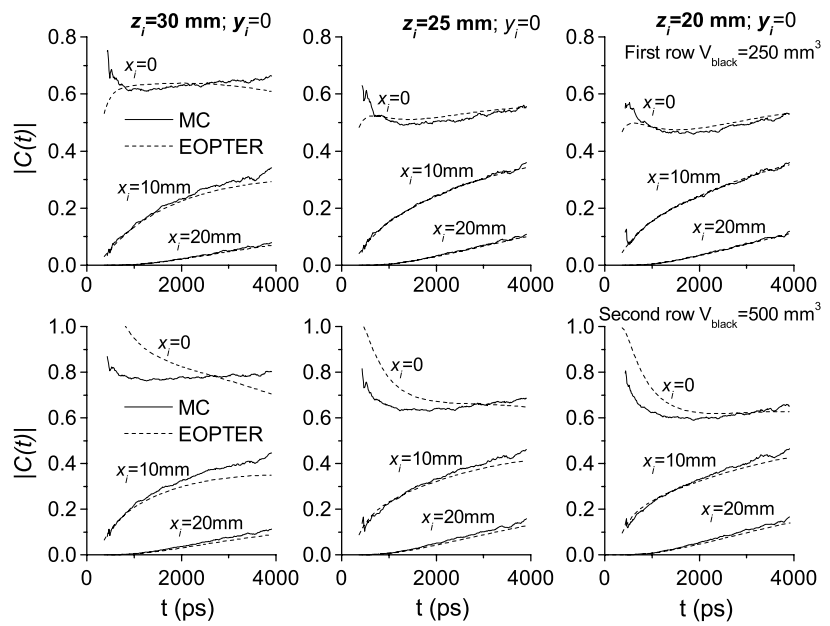

Fig. 5. Comparison of time-resolved relative transmittance contrast $|C(t)|$ obtained with MC simulations and with the EOPTER solver for a black inclusion located inside a slab $40 \mathrm{~mm}$ thick with $\mu_{s}^{\prime}=1 \mathrm{~mm}^{-1}$. The plots pertain to three arrangements of the inclusion [positions $\left.\left(x_{i}, y_{i}, z_{i}\right)\right]$.

obtained when the totally absorbing inclusion is too close to the source or the detector. The overall agreement between the EOPT and EOPTER solvers and the MC results is within about $20 \%$ and 10\%, respectively (provided we exclude the early times, i.e., those with $t>1000 \mathrm{ps}$ ). The only deviation is for the case R5 for reflectance and the case $x_{i}=0$ for transmittance.

Since for the description of totally absorbing inclusions the EOPTER solver shows the best performance, in Fig. 5 we restrict the comparisons to this solver for two larger volumes of 250 and $500 \mathrm{~mm}^{3}$. The purpose of this set of comparisons is to check the performance of the EOPTER solver for higher relative perturbation. Figure 5 shows the plot of relative temporal contrast $|C(t)|$ for the same optical properties and geometry as in Fig. 3. The EOPTER solver is shown to be, except the case $x_{i}=\overline{0}, z_{i}=30 \mathrm{~mm}$, in good agreement with the results of MC simulations. Limiting the analysis to the cases $z_{i}=20$ and $25 \mathrm{~mm}$, we have differences within about $10 \%$. A worse agreement is obtained for situations with shorter distances between the inclusions and the detector.

\section{B. CW Domain}

We propose comparisons between $\mathrm{MC}$ results and the EOPT, EOPTER, and BAER solvers for the reflectance and transmittance configurations considered in Figs. 3 and 4 where a CW source replaces the $\delta(t)$ function. Figures $\overline{6}$ (reflectance from a semi-infinite medium) and $\underline{7}$ (transmittance through a slab $4 \mathrm{~cm}$ thick) show a comparison of relative $\mathrm{CW}$ contrast $C_{\mathrm{CW}}$ for three different totally absorbing inclusions. The volumes and depths of the inclusions were the same as those Figs. 3 and 4 , and with a medium with $\mu_{s}^{\prime}=1 \mathrm{~mm}^{-1}$, $\mu_{a}=0.01 \mathrm{~mm}^{-1}$, and refractive index equal to 1.33 ; no mismatch with the external was considered. The source is at $(0,0,0)$, whereas the detector is at $(30,0,0) \mathrm{mm}$ for Fig. 6 and at $(0,0,40) \mathrm{mm}$ for Fig. 7 . The plots display the relative contrast $\left|C_{\mathrm{CW}}\right|$ obtained with the EOPT solver (thin solid curves), the EOPTER solver (dotted curves), the BAER solver (thick solid curves), and with MC simulations (open circles) versus the $x_{i}$ position of the black defect with $y_{i}=0$. The EOPT solver is calculated assuming a value of
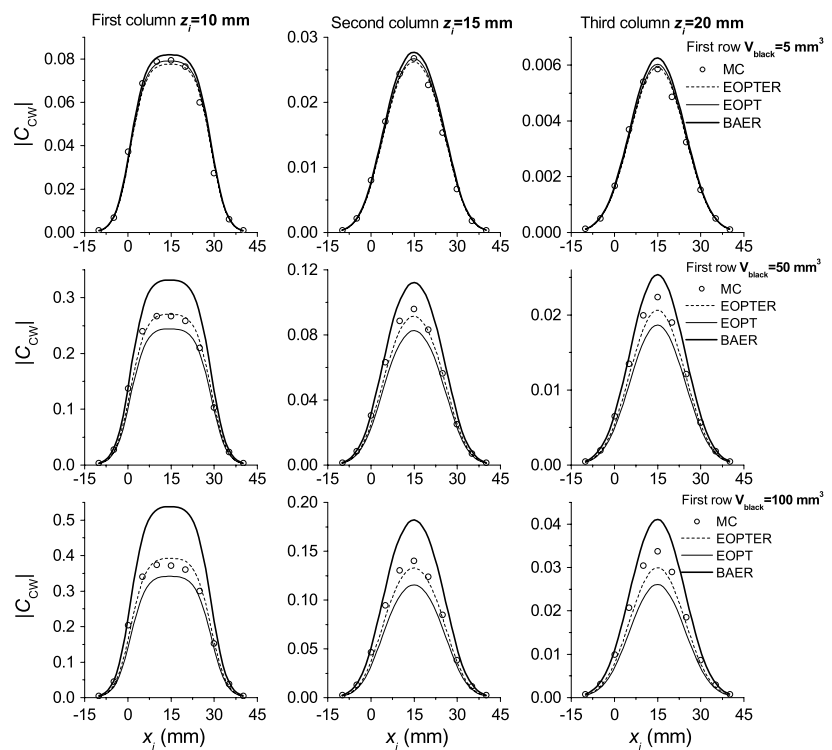

Fig. 6. Comparison of $\mathrm{CW}$ reflectance contrast obtained with $\mathrm{MC}$ simulations and with the EOPT solver, the EOPTER solver, and the BAER solver for a black inclusion located inside a semi-infinite medium with $\mu_{s}^{\prime}=1 \mathrm{~mm}^{-1}$ and $\mu_{a}=0.01 \mathrm{~mm}^{-1}$.

$\Delta \mu_{a}=10 \mathrm{~mm}^{-1}$, which practically simulates a totally absorbing inclusion. Similarly to the time domain, we note that the best agreement is obtained for the EOPTER solver. The agreement between the EOPTER solver and MC simulations is within $10 \%$. A worse behavior is obtained for the EOPT solver, which shows agreement within $20 \%$ with the MC simulations. Lastly, we have the comparisons with the BAER solver, which shows worst agreement with the MC simulations (differences up to about $30 \%-40 \%$ ) but still acceptable.

In order to have an overview of the performance of the EOPT solver for any $\Delta \mu_{a}$, Fig. $\underline{8}$ shows the plot of relative
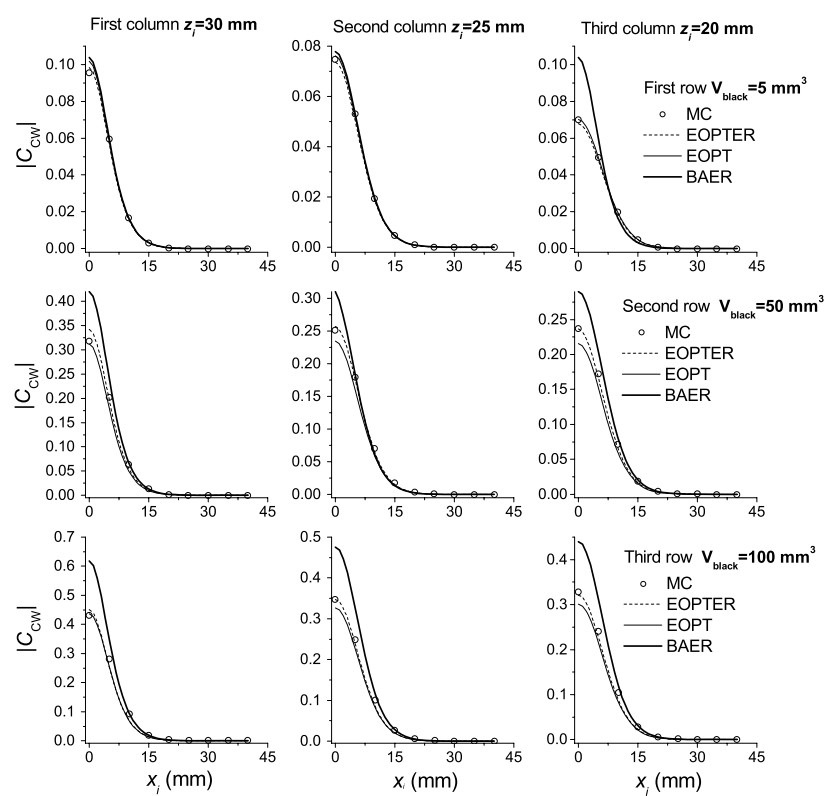

Fig. 7. Comparison of CW transmittance contrast obtained with MC simulations and with the EOPT solver, the EOPTER solver, and the BAER solver for a black inclusion located inside a slab $40 \mathrm{~mm}$ thick with $\mu_{s}^{\prime}=1 \mathrm{~mm}^{-1}$ and $\mu_{a}=0.01 \mathrm{~mm}^{-1}$. 


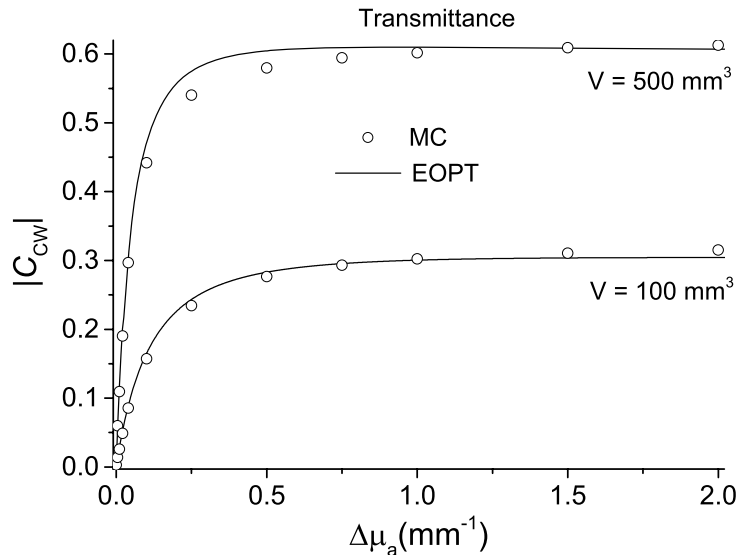

Fig. 8. Comparison of $\mathrm{CW}$ transmittance contrast for an inclusion with finite $\Delta \mu_{a}$ contrast obtained with MC simulations and with the EOPT solver for a slab $40 \mathrm{~mm}$ thick with $\mu_{s}^{\prime}=1 \mathrm{~mm}^{-1}$ and $\mu_{a}=0.01 \mathrm{~mm}^{-1}$

contrast $C_{\mathrm{CW}}$ for transmittance through a slab $4 \mathrm{~cm}$ thick obtained with the EOPT solver and with MC simulations versus variation of absorption $\Delta \mu_{a}$ between the inclusion and the background. The range of values of $\Delta \mu_{a}$ is up to saturation, which starts at about $\Delta \mu_{a}=2 \mathrm{~mm}^{-1}$. The data have been plotted for volumes of 100 and $500 \mathrm{~mm}^{3}$ for the defect placed at depth $z_{i}=20 \mathrm{~mm}, x_{i}=y_{i}=0$. A medium with $\mu_{s}^{\prime}=1 \mathrm{~mm}^{-1}$, $\mu_{a}=0.01 \mathrm{~mm}^{-1}$, and with no refractive index mismatch with the external was considered. The source is at $(0,0,0)$ and the detector at $(0,0,40) \mathrm{mm}$. The agreement between the EOPT solver and MC simulations is within about $10 \%$ for the volume of $500 \mathrm{~mm}^{3}$ and about $5 \%$ for for the volume of $100 \mathrm{~mm}^{3}$.

\section{DISCUSSION AND CONCLUSIONS}

In the comparisons presented between the EOPT, EOPTER, and BAER solvers with the results of MC simulations, we have observed that when dealing with totally absorbing inclusions, worst agreement is obtained when the position of the inclusion is close to the source and the detector. Since all the solvers used make use of the DE, we argue that the origin of this behavior is likely due to the break down of the simplifying assumptions of the diffusion approximation. Second, we have observed that in the presence of totally absorbing inclusions, the performance of the EOPTER solver is largely better than that of the EOPT solver. Thus, the ER improves the description of photon migration when applied to the EOPT. Practically, the ER improves the convergence property and the performance of the eighth-order solution. On this ground, we really expect that such a benefit of the ER is independent of the forward solver used in conjunction with this relation. As a methodological proposal, the ER can therefore be used to implement any forward solver in the calculation of the perturbation produced by a totally absorbing object. The examples produced for the BAER solver are paradigmatic in this sense. The Born approximation, which otherwise could not be used at all with totally absorbing inclusions, can be implemented together with the ER and the accuracy achievable with this solver is still acceptable for the CW domain.

About the lower accuracy that is sometimes observed for the EOPTER solver for time-domain transmittance at early times with the inclusion on-axis (Figs. 4 and 5) and in general, when the inclusion is close to the source and the detector, we can express some possible explanations. First, we notice that the ER holds whenever the photon paths can statistically cover both the totally absorbing volume and its corresponding equivalent volume. This condition is likely to fail in the conditions mentioned since the photon paths in these situations are preferentially confined inside a narrower volume compared with the equivalent volume related to the ER and this fact affects the applicability of the ER. Second, we notice that at early times and close to the source and the detector, the diffusion approximation, on which it is also based partially, the EOPTER solver, may fail. Actually, the lower accuracy at early times regards the points around the ballistic peak where the DE does not hold. Further information about the validity of the ER that may help to understand the validity of the EOPTER solver can be found in [8] where the ER is extensively studied by means of MC simulations.

The novelty of this paper is manifold. First, we have critically reviewed the literature where several forward solvers have been proposed for describing the effects of absorbing perturbations: the limitations of previously existing theories have been addressed. Second, we have improved further the solution of a heuristic perturbative approach [16-19] to the DE. Third, we have proposed a methodology that exploits the ER to reformulate the forward problem of a black or highly absorbing inclusion in terms of weaker ones with a re-scaled volume.

We have critically discussed the usefulness of the theory behind the so-called banana-shape regions [6] , which, despite its "notoriety," has found no real applications in the field of NIRS and DOT. The exact meaning of using a "small" totally absorbing object to "probe" a diffusive light field is to provide the probability of a photon detected to intersect (or pass through) the region where the object is located. However, even if we could calculate this probability, we could not reconstruct the perturbation caused by a real defect having a finite absorption contrast, which is more relevant for practical applications. There is also no evidence that knowing the probability of a photon detected to intersect a region (by using a "small" black sphere) we could use it to estimate the probability of given photon paths that connect the source and the detector (as it is hinted in [6]). In fact, for estimating this probability of photon paths, we need to know the probability of multiple events (i.e., the probability of a photon detected to visit multiple points), and therefore, other approaches (like the path integral) would be necessary. We argue that no exact expressions of the probability involving the power subtracted by a black sphere has been provided in the literature, and also that the expressions that can be derived within the DE cannot provide the correct estimates of this probability as has been shown in Section 2. We have shown this point by using a nonabsorbing diffusive medium where the DE reduces to the Poisson equation and exact solutions are easily found. We have discussed possible reasons of the failure of the DE to describe light propagation in the presence of a black object. We have pinpointed the relationship between the theory proposed for the banana-shape regions theory [6] and the Born approximation. We have stated that if one follows the reasoning of the authors, then it is possible to derive the so-called "banana-shape regions" in any bounded geometry by knowing the mean path length in the small region occupied by a probing black object. Therefore, the banana-shape approach is a 
first-order perturbation approach; we note that this observation can be linked to our recently proposed "ER," and also to some of the results shown in this paper where we have applied this relation and the Born approximation for calculating the perturbation induced by a black sphere.

We have presented two forward solvers, EOPT and EOPTER, and a new methodology for description of photon migration in the presence of highly or totally absorbing inclusions embedded in diffusive media in both time and CW domains. We have improved further the solution of a heuristic perturbative approach [16-19] based on a higher order perturbation theory and the Padé approximants by developing the expansion up to the eighth order. We have also proposed the use of the ER as a way to reformulate the problem of highly or totally absorbing inclusions in terms of milder ones with a re-scaled volume. This methodology is quite general and can be applied to different forward models. By using MC simulations, we have shown that the EOPTER solver can model totally absorbing inclusions with an error smaller than about 10\%, whereas the EOPT solver shows an error smaller than about $20 \%$. Looking at Fig. 1, we can conclude by noting that the performances of the previous theories, like the banana-shape regions theory, are considerably worse than those of the proposed forward solvers.

\section{ACKNOWLEDGMENTS}

The research leading to these results has partially received support from the European Community's Seventh Framework Programme (nEUROPt Project, grant agreement no. 201076 and LASERLAB-EUROPE Project, grant agreement no. 284464), NIH grant R01-CA154774, and NSF Award IIS 1065154

\section{REFERENCES}

1. T. Durduran, R. Choe, W. B. Baker, and A. G. Yodh, "Diffuse optics for tissue monitoring and tomography," Rep. Prog. Phys. 73, 076701 (2010).

2. A. Torricelli, L. Spinelli, A. Pifferi, P. Taroni, R. Cubeddu, and G. Danesini, "Use of a nonlinear perturbation approach for in vivo breast lesion characterization by multiwavelength time-resolved optical mammography," Opt. Express 11, 853-867 (2003).

3. R. Choe, A. Corlu, K. Lee, T. Durduran, S. D. Konecky, M. Grosicka-Koptyra, S. R. Arridge, B. J. Czerniecki, D. L. Fraker, A. DeMichele, B. Chance, M. A. Rosen, and A. G. Yodh, "Diffuse optical tomography of breast cancer during neoadjuvant chemotherapy: a case study with comparison to MRI," Breast Cancer Res. Treat. 32, 1128-1139 (2005).

4. M. R. Stankovic, D. Maulik, W. Rosenfeld, P. G. Stubblefield, A. D. Kofinas, E. Gratton, M. A. Franceschini, S. Fantini, and D. M. Hueber, "Role of frequency domain optical spectroscopy in the detection of neonatal brain hemorrhage - a newborn piglet study," J. Matern. Fetal Med. 9, 142-149 (2000).

5. A. Amelink, T. Christiaanse, and H. J. C. M. Sterenborg, "Effect of hemoglobin extinction spectra on optical spectroscopic measurements of blood oxygen saturation," Opt. Lett. 34, 1525-1527 (2009).
6. S. Feng, F. Zeng, and B. Chance, "Photon migration in the presence of a single defect: a perturbation analysis," Appl. Opt. 34, 3826-3837 (1995).

7. X. D. Zhu, S. Wei, S. C. Feng, and B. Chance, "Analysis of a diffuse-photon-density wave in multiple-scattering media in the presence of a small spherical object," J. Opt. Soc. Am. A 13, 494-499 (1996).

8. F. Martelli, A. Pifferi, D. Contini, L. Spinelli, A. Torricelli, H. Wabnitz, R. Macdonald, A. Sassaroli, and G. Zaccanti, "Phantoms for diffuse optical imaging based on totally absorbing objects. Part I: basic concepts," J. Biomed. Opt. 18, 066014 (2013).

9. A. Wabnitz, A. Jelzow, M. Mazurenka, O. Steinkellner, R. Macdonald, A. Pifferi, A. Torricelli, D. Contini, L. Zucchelli, R. Cubeddu, L. Spinelli, D. Milej, N. Zolek, M. Kacprzak, P. Sawosz, A. Liebert, S. Magazov, J. Hebden, F. Martelli, P. Di Ninni, and G. Zaccanti, "Performance assessment of time-domain optical brain imagers: a multi-laboratory study," Proc. SPIE 8583, 85830L (2013).

10. D. A. Boas, M. A. O'Leary, B. Chance, and A. G. Yodh, "Scattering of diffuse photon density waves by spherical inhomogeneities within turbid media: analytic solution and applications," Proc. Natl. Acad. Sci. USA 91, 4887-4891 (1994).

11. M. R. Ostermeyer and S. L. Jacques, "Perturbation theory for diffuse light transport in complex biological tissues," J. Opt. Soc. Am. A 14, 255-261 (1997).

12. S. Carraresi, T. S. M. Shatir, F. Martelli, and G. Zaccanti, "Accuracy of a perturbation model to predict the effect of scattering and absorbing inhomogeneities on photon migration," Appl. Opt. 40, 4622-4632 (2001).

13. L. Spinelli, A. Torricelli, A. Pifferi, P. Taroni, and R. Cubeddu, "Experimental test of a perturbation model for time-resolved imaging in diffusive media," Appl. Opt. 42, 3145-3153 (2003).

14. J. C. J. Paasschens and G. W. 't Hooft, "Influence of boundaries on the imaging of objects in turbid media," J. Opt. Soc. Am. A 15, 1797-1812 (1998).

15. F. Martelli, S. Del Bianco, A. Ismaelli, and G. Zaccanti, Light Propagation through Biological Tissue and Other Diffusive Media (SPIE, 2010).

16. A. Sassaroli, F. Martelli, and S. Fantini, "Perturbation theory for the diffusion equation by use of the moments of the generalized temporal point-spread function. I. Theory," J. Opt. Soc. Am. A 23, 2105-2118 (2006).

17. A. Sassaroli, F. Martelli, and S. Fantini, "Perturbation theory for the diffusion equation by use of the moments of the generalized temporal point-spread function. II. Continuous-wave results," J. Opt. Soc. Am. A 23, 2119-2131 (2006).

18. A. Sassaroli, F. Martelli, and S. Fantini, "Higher-order perturbation theory for the diffusion equation in heterogeneous media: application to layered and slab geometries," Appl. Opt. 48, D62-D73 (2009).

19. A. Sassaroli, F. Martelli, and S. Fantini, "Perturbation theory for the diffusion equation by use of the moments of the generalized temporal point-spread function. III. Frequency-domain and timedomain results," J. Opt. Soc. Am. A 27, 1723-1742 (2010).

20. R. Graaff and K. Rinzema, "Practical improvements on photon diffusion theory: application to isotropic scattering," Phys. Med. Biol. 46, 3043-3050 (2001).

21. J. D. Jackson, Classical Electrodynamics (Wiley, 1962).

22. J. C. J. Paasschens, "Solution of the time-dependent Boltzmann equation," Phys. Rev. E 56, 1135-1141 (1997).

23. A. Sassaroli, C. Blumetti, F. Martelli, L. Alianelli, D. Contini, A. Ismaelli, and G. Zaccanti, "Monte Carlo procedure for investigating light propagation and imaging of highly scattering media," Appl. Opt. 37, 7392-7400 (1998). 